Rev. Mat. IBeroamericana 21 (2005), no. 1, 1-24

\title{
The Cauchy problem for viscous shallow water equations
}

\section{Weike Wang and Chao-Jiang $\mathrm{Xu}$}

\begin{abstract}
In this paper we study the Cauchy problem for viscous shallow water equations. We work in the Sobolev spaces of index $s>2$ to obtain local solutions for any initial data, and global solutions for small initial data.
\end{abstract}

\section{Introduction}

We consider in this work the following Cauchy problems for viscous shallow water equations:

$$
\begin{gathered}
h\left(u_{t}+(u \cdot \nabla) u\right)-\nu \nabla \cdot(h \nabla u)+h \nabla h=0, \\
h_{t}+\operatorname{div}(h u)=0, \\
\left.u\right|_{t=0}=u_{0},\left.\quad h\right|_{t=0}=h_{0} ;
\end{gathered}
$$

where $h(x, t)$ is the height of fluid surface, $u(x, t)=\left(u^{1}(x, t), u^{2}(x, t)\right)^{t}$ is the horizontal velocity field, $x=\left(x_{1}, x_{2}\right) \in \mathbb{R}^{2}$ and $0<\nu<1$ is the viscous coefficient.

These equations form a quasi-linear hyperbolic-parabolic system. For the initial data $h_{0}(x)$, we shall consider small perturbations of some positive constant $\bar{h}_{0}$. And we will study the Cauchy problem (1.1)-(1.3) in Sobolev function spaces. The main theorem of this paper is the following :

Theorem 1.1 Let $s>0, u_{0}, h_{0}-\bar{h}_{0} \in H^{2+s}\left(\mathbb{R}^{2}\right),\left\|h_{0}-\bar{h}_{0}\right\|_{H^{2+s}}<<\bar{h}_{0}$. Then there exist a positive time $T$ and a unique solution $(u, h)$ of Cauchy problem (1.1)-(1.3) such that

$$
u, h-\bar{h}_{0} \in L^{\infty}\left([0, T] ; H^{2+s}\right), \quad \nabla u \in L^{2}\left([0, T] ; H^{2+s}\right) .
$$

Furthermore, there exists a constant c such that if $\left\|h_{0}-\bar{h}_{0}\right\|_{H^{2+s}}+\left\|u_{0}\right\|_{H^{2+s}} \leq c$ then we can choose $T=+\infty$. 
In [2], it was obtained the local existence and uniqueness of classical solutions to the Cauchy-Dirichlet problem for the shallow water equations using Lagrangian coordinates and Hölder space estimates with initial data in $C^{2+\alpha}$. Kloeden [5] and Sundbye [10] proved global existence and uniqueness of classical solutions to the Cauchy-Dirichlet problem using Sobolev space estimates and the energy method of Matsumura and Nishida [7, 8, 9]. Sundbye [11] proved also the existence and uniqueness of classical solutions to the Cauchy problem using the method of $[7,8,9]$. However, these results only consider the case of small initial data. In general, the problem of existence of solutions for large initial data is difficult, because its strong non-linear nature. In this paper, we use the Littlewood-Paley decomposition theory (see $[1,3])$ for Sobolev spaces to obtain a losing energy estimate in $H^{s+2}$ for any $s>0$, which allows us to get the local existence of solution for all initial data. Moreover, we also improve the global existence of solution and regularity for small initial data. From this result of global existence, we can obtain some decay estimate as in $[6,12]$ with the method of Green function but, for brevity, we leave it for the future.

The structure of the paper is the following:

In section 2 we recall Littlewood-Paley theory for Sobolev spaces. In section 3 we prove the first part of the main theorem: local existence of solution for all size of the initial data. In section 4 we prove the global existence of solution for small initial data. Finally, in section 5 we prove the losing energy estimates for the nonlinear terms.

\section{Littlewood-Paley theory}

Let us recall Sobolev spaces and Littlewood-Paley theory (see, for example, Bony [1] and Chemin [3]). There exist functions $\varphi$ and $\psi$ in $C_{0}^{\infty}\left(\mathbb{R}^{d}\right)$ such that Supp $\varphi \subset \mathcal{C}=\{\xi ; 5 / 6 \leq|\xi| \leq 12 / 5\}$, Supp $\psi \subset B=\{|\xi| \leq 2\}$,

$$
\forall \xi \in \mathbb{R}^{d} \backslash\{0\}, \sum_{j \in \mathbb{Z}} \varphi\left(2^{-j} \xi\right)=1 \text { and } \forall \xi \in \mathbb{R}^{d}, \psi(\xi)+\sum_{j \in \mathbb{N}} \varphi\left(2^{-j} \xi\right)=1 .
$$

Let us note that if $\left|j-j^{\prime}\right| \geq 2$, then $\operatorname{Supp} \varphi\left(2^{-j} \cdot\right) \cap \operatorname{Supp} \varphi\left(2^{-j^{\prime}} \cdot\right)=\emptyset$. We define the following operators of localization in Fourier space

$$
\dot{\Delta}_{j} u=\mathcal{F}^{-1}\left(\varphi\left(2^{-j} \cdot\right) \hat{u}(\cdot)\right)=2^{j d} \int_{\mathbb{R}^{d}} f\left(2^{j} y\right) u(x-y) d y, \text { for } j \in \mathbb{Z}
$$

and

$$
\Delta_{-1} u=\mathcal{F}^{-1}(\psi(\cdot) \hat{u}(\cdot)), \quad \Delta_{j}=\dot{\Delta}_{j}, \text { for } j \in \mathbb{N}
$$


where $\hat{u}$ denote the Fourier transformation of $u$, and $f=\mathcal{F}^{-1}(\varphi)$. So that for $u \in \mathcal{S}^{\prime}$, we have that $\Delta_{j} u, \Delta_{-1} u \in C^{\infty} \cap L^{2}$. Then the Sobolev space is defined, for $s \in \mathbb{R}$, as follows:

$$
H^{s}\left(\mathbb{R}^{2}\right)=\left\{u \in \mathcal{S}^{\prime}\left(\mathbb{R}^{2}\right) ;\|u\|_{H^{s}}^{2}=\sum_{j=-1}^{\infty} 2^{2 j s}\left\|\Delta_{j} u\right\|_{L^{2}}^{2}<+\infty\right\} .
$$

In the low vertical frequencies estimates, we have to use the homogeneous Sobolev spaces,

$$
\dot{H}^{s}\left(\mathbb{R}^{2}\right)=\left\{u \in \mathcal{S}^{\prime}\left(\mathbb{R}^{2}\right) ;\|u\|_{\dot{H}^{s}}^{2}=\sum_{j \in \mathbb{Z}} 2^{2 j s}\left\|\dot{\Delta}_{j} u\right\|_{L^{2}}^{2}<+\infty\right\} .
$$

For $d=2$, we have that $H^{2+s}\left(\mathbb{R}^{2}\right) \subset L^{\infty}\left(\mathbb{R}^{2}\right)$ for any $s>-1$, and

$$
\|f\|_{L^{\infty}\left(\mathbb{R}^{2}\right)} \leq C_{s}\|f\|_{H^{2+s}\left(\mathbb{R}^{2}\right)}
$$

where $C_{s}$ is Sobolev constant in $\mathbb{R}^{2}$. We have also that, for any $q \geq 0$,

$$
\left\|\Delta_{q} f\right\|_{L^{\infty}} \leq C\left\|\nabla\left(\Delta_{q} f\right)\right\|_{L^{2}}
$$

and

$$
\left\|\Delta_{q} f\right\|_{L^{2}} \leq C 2^{-q}\left\|\nabla\left(\Delta_{q} f\right)\right\|_{L^{2}} .
$$

We set $S_{q}(u)=\sum_{-1 \leq p \leq q-2} \Delta_{p} u$, then $S_{q}: H^{s} \rightarrow H^{+\infty}$,

$$
\Delta_{p}\left(S_{q}(u) \Delta_{q} u\right)=0 \text {, if }|p-q| \geq 4 ; \text { and }\left\|S_{q}(\nabla u)\right\|_{L^{\infty}} \leq 2^{q}\left\|S_{q} u\right\|_{L^{\infty}}
$$

For the product of two functions, we have the decomposition:

$$
\begin{aligned}
u v & =\sum_{q} S_{q-1}(u) \Delta_{q} v+\sum_{q} S_{q-1}(v) \Delta_{q} u+\sum_{|p-q|<2} \Delta_{p} u \Delta_{q} v \\
& =T_{u} v+T_{v} u+R(u, v)
\end{aligned}
$$

where $T_{u}$ is a linear operator. We have:

if $u \in L^{\infty}$, then for all $s \in \mathbb{R}$,

$$
\left\|T_{u}\right\|_{\mathcal{L}\left(H^{s}, H^{s}\right)} \leq C\|u\|_{L^{\infty}}
$$

if $u \in H^{\tau}, \tau<d / 2$, then for all $s \in \mathbb{R}$,

$$
\left\|T_{u}\right\|_{\mathcal{L}\left(H^{s}, H^{s+\tau-d / 2}\right)} \leq C\|u\|_{H^{\tau}}
$$

if $u \in H^{s_{1}}, v \in H^{s_{2}}, s_{1}+s_{2}-d / 2>0$, then

$$
\|R(u, v)\|_{H^{s_{1}+s_{2}-d / 2}} \leq C\|u\|_{H^{s_{1}}}\|v\|_{H^{s_{2}}} .
$$


For the nonlinear composition, if $F \in C^{\infty}(I)$ such that $F(0)=0, u \in$ $H^{\tau}\left(\mathbb{R}^{2}\right), \tau>1$ with $u(x) \in I$ for all $x \in \mathbb{R}^{2}$, then there exists a function of one variable $B_{0}$ depending only on $\tau, F, I$ such that

$$
\|F(u)\|_{H^{\tau}} \leq B_{0}\left(\|u\|_{L^{\infty}}\right)\|u\|_{H^{\tau}} .
$$

In our equation, we have the products of 3 functions, so that we need the following precise estimates:

$$
\begin{aligned}
\left|(a b, c)_{L^{2}}\right| & \leq C\|a\|_{L^{\infty}}\|b\|_{L^{2}}\|c\|_{L^{2}}, \\
\left|(a b, c)_{L^{2}}\right| & \leq C\|a\|_{\dot{H}^{1 / 2}}\|b\|_{L^{2}}\|c\|_{\dot{H}^{1 / 2}} \\
\|a\|_{\dot{H}^{1 / 2}}^{2} & \leq\|a\|_{L^{2}}\|\nabla a\|_{L^{2}} .
\end{aligned}
$$

For the detail of those results, we send to the reference [3].

In the proof of main theorem, we need to estimate the nonlinear term in the equations, using the so-called "Losing energy estimates".

Lemma 2.1 Let $\tau>1$ and $-1 \leq k<+\infty$, then there exists $C_{0}>0$ such that for all $v, \nabla v, g, \nabla g \in H^{\tau}$, we have

$$
\left|\int_{\mathbb{R}^{2}} \Delta_{k}((v \cdot \nabla) g) \Delta_{k} g d x\right| \leq C_{0} d_{k}^{2} 2^{-2 k \tau}\|v\|_{H^{\tau+1}}\|g\|_{H^{\tau}}^{2},
$$

with $\left\{d_{k}\right\} \in \ell^{2}$ and $\left\|\left\{d_{k}\right\}\right\|_{\ell^{2}} \leq 1$.

Lemma 2.2 (a) Let $\tau>2$ and $-1 \leq k<+\infty$, then there exists $C_{0}>0$ such that for all $f, v, g, u, \nabla u \in H^{\tau}$, with $\|g\|_{L^{\infty}} \leq 1 / 4$, we have

$$
\left|\int_{\mathbb{R}^{2}} \Delta_{k}\left(\frac{\nabla f}{1+g} \nabla v\right) \Delta_{k} u d x\right| \leq C_{0} d_{k}^{2} 2^{-2 k \tau}\|f\|_{H^{\tau}}\|v\|_{H^{\tau}}\left(1+\|g\|_{H^{\tau}}\right)\|u\|_{H^{\tau+1}}
$$

where $\left\|\left\{d_{k}\right\}\right\|_{\ell^{2}} \leq 1$.

(b) Let $1<\tau<2$ and $-1 \leq k<+\infty$, then there exists $C_{0}>0$ such that for all $f, g, u, \nabla u, v, \nabla v \in H^{\tau}$, with $\|g\|_{L^{\infty}} \leq 1 / 4$, we have

$$
\left|\int_{\mathbb{R}^{2}} \Delta_{k}\left(\frac{\nabla f}{1+g} \nabla v\right) \Delta_{k} u d x\right| \leq C_{0} d_{k}^{2} 2^{-2 k \tau}\|f\|_{H^{\tau}}\left(1+\|g\|_{H^{\tau}}\right) U_{\tau}(u, v),
$$

with $\left\|\left\{d_{k}\right\}\right\|_{\ell^{2}} \leq 1$, and

$$
U_{\tau}(u, v)=:\|\nabla v\|_{L^{\infty}}\|u\|_{H^{\tau+1}}+\|\nabla v\|_{H^{\tau}}\left(\|\nabla u\|_{H^{1}}+\|u\|_{H^{\tau}}\right) .
$$


Lemma 2.3 (a) Let $\tau>2$ and $-1 \leq k<+\infty$, then there exists $C_{0}>0$ such that for all $f, v, u, \nabla u, g_{1}, g_{2} \in H^{\tau}$, with $\left\|g_{1}\right\|_{L^{\infty}},\left\|g_{2}\right\|_{L^{\infty}} \leq 1 / 4$, we have

$$
\begin{aligned}
\mid \int_{\mathbb{R}^{2}} \Delta_{k}\left(\frac{\left(g_{1}-g_{2}\right)}{\left(1+g_{1}\right)\left(1+g_{2}\right)}\right. & \nabla f \nabla v) \Delta_{k} u d x \mid \\
& \leq C_{0} d_{k}^{2} 2^{-2 k \tau}\|f\|_{H^{\tau}}\|v\|_{H^{\tau}}\left\|g_{1}-g_{2}\right\|_{H^{\tau}}\|u\|_{H^{\tau+1}},
\end{aligned}
$$

with $\left\|\left\{d_{k}\right\}\right\|_{\ell^{2}} \leq 1$.

(b) Let $1<\tau<2$ and $-1 \leq k<+\infty$, then there exists $C_{0}>0$ such that for all $f, v, g_{1}, g_{2}, u, \nabla u, v, \nabla v \in H^{\tau}$, with $\left\|g_{1}\right\|_{L^{\infty}},\left\|g_{2}\right\|_{L^{\infty}} \leq 1 / 4$, we have

$$
\begin{aligned}
\mid \int_{\mathbb{R}^{2}} \Delta_{k}\left(\frac{\left(g_{1}-g_{2}\right)}{\left(1+g_{1}\right)\left(1+g_{2}\right)}\right. & \nabla f \nabla v) \Delta_{k} u d x \mid \\
& \leq C_{0} d_{k}^{2} 2^{-2 k \tau}\|f\|_{H^{\tau}}\left\|g_{1}-g_{2}\right\|_{H^{\tau}} U_{\tau}(u, v),
\end{aligned}
$$

with $\left\|\left\{d_{k}\right\}\right\|_{\ell^{2}} \leq 1$, and $U_{\tau}(u, v)$ is as in Lemma 2.2, (b).

In the proof of existence of global solutions, we need the following high vertical frequencies estimates:

Lemma 2.4 Let $\tau>0$, then there exists $M>0, C_{0}>0$ such that for all $h, u, v, \nabla h, \nabla u \in H^{\tau}, M \leq k<+\infty$, with $\|h\|_{L^{\infty}} \leq 1 / 4$, we have

$$
\begin{aligned}
& \mid \int_{\mathbb{R}^{2}} \Delta_{k}\left(\frac{1}{1+h}\right.\nabla h \nabla u) \Delta_{k} v d x \mid \\
& \leq C_{0} d_{k}^{2} 2^{-2 k \tau}\left(1+\|h\|_{H^{\tau+1}}\right)\|\Delta u\|_{H^{\tau}}\|\nabla h\|_{H^{\tau}}\|v\|_{H^{\tau}},
\end{aligned}
$$

with $\left\|\left\{d_{k}\right\}\right\|_{\ell^{2}} \leq 1$.

Lemma 2.5 Let $\tau>0$, then there exists $M>0, C_{0}>0$ such that for all $h \in H^{\tau+1}$ with $\|h\|_{L^{\infty}} \leq 1 / 4$, and $u \in H^{\tau+2}, M \leq k<+\infty$, we have

$$
\begin{aligned}
\mid \int_{\mathbb{R}^{2}} \Delta_{k}(\operatorname{div}(h u)) & \Delta_{k}(\Delta h) d x \mid \\
& \leq C_{0} d_{k}^{2} 2^{-2 k \tau}\|\nabla h\|_{H^{\tau}}\left(\|\nabla h\|_{H^{\tau}}^{2}+\|\nabla u\|_{H^{\tau+1}}^{2}\right),
\end{aligned}
$$

with $\left\|\left\{d_{k}\right\}\right\|_{\ell^{2}} \leq 1$.

We will prove these five lemmas in the last section. 


\section{The local existence of solution}

In order to study the local existence of solution, we define the function set $(f, g) \in \mathcal{X}\left(\left[t_{1}, t_{2}\right], \sigma, E_{1}, E_{2}\right)$ if

$$
(f, g) \in L^{\infty}\left(\left[t_{1}, t_{2}\right], H^{\sigma}\left(\mathbb{R}^{2}\right)\right), \quad \nabla f \in L^{2}\left(\left[t_{1}, t_{2}\right], H^{\sigma}\left(\mathbb{R}^{2}\right)\right)
$$

and

$$
\begin{gathered}
\|f\|_{L^{\infty}\left(\left[t_{1}, t_{2}\right], H^{\sigma}\left(\mathbb{R}^{2}\right)\right)}^{2}+\nu\|\nabla f\|_{L^{2}\left(\left[t_{1}, t_{2}\right], H^{\sigma}\left(\mathbb{R}^{2}\right)\right)}^{2} \leq E_{1}^{2} \\
\|g\|_{L^{\infty}\left(\left[t_{1}, t_{2}\right], H^{\sigma}\left(\mathbb{R}^{2}\right)\right)} \leq E_{2} .
\end{gathered}
$$

The main result of this section is the following local existence theorem for any initial data:

Theorem 3.1 Let $s>0,\left(u_{0}, h_{0}-\bar{h}_{0}\right) \in H^{s+2}\left(\mathbb{R}^{2}\right)$ with $\left\|h_{0}-\bar{h}_{0}\right\|_{H^{2+s}} \leq \frac{\bar{h}_{0}}{4 C_{s}}$, then there exist a positive time $T$ and a solution

$$
\left(u, h-\bar{h}_{0}\right) \in \mathcal{X}\left([0, T], s+2, E_{1}, E_{2}\right)
$$

for the Cauchy problem (1.1)-(1.3). Here

$$
E_{1}=2\left\|u_{0}\right\|_{H^{s+2}}, \quad E_{2}=2\left\|h_{0}-\bar{h}_{0}\right\|_{H^{s+2}}
$$

and $C_{s}$ is the Sobolev constant.

For the sake of convenience, we take $\bar{h}_{0}=1$. Changing $h$ by $1+h$ in (1.1)-(1.3), we have

$$
\begin{gathered}
u_{t}+(u \cdot \nabla) u-\nu \frac{\nabla \cdot((1+h) \nabla u)}{1+h}+\nabla h=0, \\
h_{t}+\operatorname{div} u+\operatorname{div}(h u)=0, \\
u(x, 0)=u_{0}(x), h(x, 0)=h_{0}(x) .
\end{gathered}
$$

We suppose now that $h_{0} \in H^{s+2}\left(\mathbb{R}^{2}\right),\left\|h_{0}\right\|_{H^{2+s}} \leq \frac{1}{4 C_{s}}$, and $E_{1}=2\left\|u_{0}\right\|_{H^{s+2}}$, $E_{2}=2\left\|h_{0}\right\|_{H^{s+2}}$.

The proof of Theorem 3.1 involves the method of successive approximations. Let us define the sequence $\left\{u_{n}, h_{n}\right\}$ by the following linear systems:

$$
\begin{gathered}
\left(u_{1}, h_{1}\right)=S_{2}\left(u_{0}, h_{0}\right), \\
\partial_{t} u_{n+1}-\nu \triangle u_{n+1}=G_{1}\left(u_{n}, h_{n}\right), \\
\partial_{t} h_{n+1}+u_{n} \nabla h_{n+1}=G_{2}\left(u_{n}, h_{n}\right), \\
\left.\left(u_{n+1}, h_{n+1}\right)\right|_{t=0}=S_{n+2}\left(u_{0}, h_{0}\right),
\end{gathered}
$$

where

$$
\begin{aligned}
& G_{1}\left(u_{n}, h_{n}\right)=\frac{\nu}{1+h_{n}} \nabla h_{n} \nabla u_{n}-u_{n} \nabla u_{n}+\nabla h_{n} \\
& G_{2}\left(u_{n}, h_{n}\right)=-\left(1+h_{n}\right) \operatorname{div} u_{n} .
\end{aligned}
$$


Since $S_{q}$ are smooth operators, the initial data $S_{n+2}\left(u_{0}, h_{0}\right)$ are smooth functions. If $\left(u_{n}, h_{n}\right) \in \mathcal{X}\left([0, T], s+2, E_{1}, E_{2}\right)$ and smooth, we have

$$
\left\|h_{n}\right\|_{L^{\infty}} \leq C_{s}\left\|h_{n}\right\|_{H^{2+s}} \leq C_{s} E_{2}=2 C_{s}\left\|h_{0}\right\|_{H^{2+s}} \leq \frac{2 C_{s}}{4 C_{s}} \leq \frac{1}{2},
$$

then $G_{1}\left(u_{n}, h_{n}\right)$ and $G_{2}\left(u_{n}, h_{n}\right)$ are also smooth functions. Note that (3.4) is the heat equation for $u_{n+1}$, while (3.5) is the transport equation for $h_{n+1}$. Therefore, the existence of smooth solutions for the Cauchy problems (3.4)-(3.6) is evident. We denote by $P_{n}$ the application from $\left(u_{n}, h_{n}\right)$ to $\left(u_{n+1}, h_{n+1}\right)$ the solution of problem (3.4)-(3.6).

Now the proof of Theorem 3.1 consists in two steps: "Estimates for big norms" and "convergence for small norms".

\section{Estimates for big norms}

Proposition 3.1 Suppose that $\left(u_{0}, h_{0}\right) \in H^{s+2}\left(\mathbb{R}^{2}\right)$ for $s>0$ and $\left\|h_{0}\right\|_{H^{s+2}} \leq$ $\frac{1}{4 C_{s}}$, then there exists a positive time $T_{1}$ such that for any $n \in \mathbb{N}, P_{n}$ is an application from $\mathcal{X}\left(\left[0, T_{1}\right], s+2, E_{1}, E_{2}\right)$ to $\mathcal{X}\left(\left[0, T_{1}\right], s+2, E_{1}, E_{2}\right)$ for $E_{1}=2\left\|u_{0}\right\|_{H^{s+2}}, E_{2}=2\left\|h_{0}\right\|_{H^{s+2}}$.

Proof. For the sake of convenience, we suppose that $1 \leq E_{1}$ (the proof for $E_{1}<1$ is easy), and remark that $0<E_{2}<1,0<\nu<1$. We take now

$$
T_{1}=\min \left\{\left(\frac{12}{5} K\right)^{-2}, \frac{\nu E_{2}^{2}}{16 C_{0}^{2} E_{1}^{4}}\right\},
$$

where $K=\left\|\mathcal{F}^{-1}(\varphi)\right\|_{L^{1}}$. We prove the proposition by induction. Firstly, $\left(u_{1}, h_{1}\right)=S_{2}\left(u_{0}, h_{0}\right)$, then

$$
\begin{gathered}
\left\|u_{1}\right\|_{H^{s+2}} \leq\left\|u_{0}\right\|_{H^{s+2}}, \quad\left\|h_{1}\right\|_{H^{s+2}} \leq\left\|h_{0}\right\|_{H^{s+2}}, \\
\nu \int_{0}^{T_{1}}\left\|\nabla u_{1}\right\|_{H^{s+2}}^{2} d \tau \leq \nu T_{1}\left(\frac{12}{5} K\right)^{2}\left\|u_{0}\right\|_{H^{s+2}}^{2} \leq\left\|u_{0}\right\|_{H^{s+2}}^{2} .
\end{gathered}
$$

Thus $\left(u_{1}, h_{1}\right) \in \mathcal{X}\left(\left[0, T_{1}\right], s+2, E_{1}, E_{2}\right)$.

Now, we assume that $\left(u_{n}, h_{n}\right) \in \mathcal{X}\left(\left[0, T_{1}\right], s+2, E_{1}, E_{2}\right)$ is valid and prove that $P_{n}\left(u_{n}, h_{n}\right)=\left(u_{n+1}, h_{n+1}\right) \in \mathcal{X}\left(\left[0, T_{1}\right], s+2, E_{1}, E_{2}\right)$ is also valid.

Applying the operator $\Delta_{k}$ to the equations (3.4), (3.5), multiplying the first by $\Delta_{k} u_{n+1}$, and the second by $\Delta_{k} h_{n+1}$, integration over $\mathbb{R}^{2}$ yields

$$
\begin{aligned}
\partial_{t}\left\|\Delta_{k} u_{n+1}\right\|_{L^{2}}^{2}+2 \nu\left\|\nabla \Delta_{k} u_{n+1}\right\|_{L^{2}}^{2} & =2 \int_{\mathbb{R}^{2}} \Delta_{k} G_{1}\left(u_{n}, h_{n}\right) \Delta_{k} u_{n+1} d x, \\
\partial_{t}\left\|\Delta_{k} h_{n+1}\right\|_{L^{2}}^{2}-2 \int_{\mathbb{R}^{2}} \Delta_{k}\left(u_{n} \nabla h_{n+1}\right) & \Delta_{k} h_{n+1} d x \\
& =2 \int_{\mathbb{R}^{2}} \Delta_{k} G_{2}\left(u_{n}, h_{n}\right) \Delta_{k} h_{n+1} d x .
\end{aligned}
$$


8 W. Wang and C-J. Xu

Using Lemma 2.1, Lemma 2.2 (a) and hypotheses on $\left(u_{n}, h_{n}\right)$, we obtain

$$
\begin{aligned}
\partial_{t} \| & \Delta_{k} u_{n+1}\left\|_{L^{2}}^{2}+2 \nu\right\| \Delta_{k}\left(\nabla u_{n+1}\right) \|_{L^{2}}^{2} \leq C_{0} d_{k}^{2} 2^{-2 k(s+2)} \\
& \times\left(\left\|h_{n}\right\|_{H^{s+2}}\left\|\nabla u_{n+1}\right\|_{H^{s+2}}+V_{1}(t)\left(\left\|u_{n+1}\right\|_{H^{s+2}}+\left\|\nabla u_{n+1}\right\|_{H^{s+2}}\right)\right), \\
\partial_{t} \| & \Delta_{k} h_{n+1} \|_{L^{2}}^{2} \leq C_{0} d_{k}^{2} 2^{-2 k(s+2)} \\
& \times\left(\left\|u_{n}\right\|_{H^{s+2}}\left\|h_{n+1}\right\|_{H^{s+2}}^{2}+\left(1+\left\|h_{n}\right\|_{H^{s+2}}\right)\left\|\nabla u_{n}\right\|_{H^{s+2}}\left\|h_{n+1}\right\|_{H^{s+2}}\right),
\end{aligned}
$$

where

$$
V_{1}(t)=\left\|h_{n}(t)\right\|_{H^{s+2}}\left\|u_{n}(t)\right\|_{H^{s+2}}\left(1+\left\|h_{n}(t)\right\|_{H^{s+2}}\right)+\left\|u_{n}(t)\right\|_{H^{s+2}}^{2} \leq \frac{3}{4} E_{1}^{2} .
$$

Multiplying (3.7) and (3.8) by $2^{2 k(s+2)}$, and taking the sum over $k$ gives respectively

$$
\begin{aligned}
\partial_{t}\left\|u_{n+1}\right\|_{H^{s+2}}^{2}+\nu\left\|\nabla u_{n+1}\right\|_{H^{s+2}}^{2} & \leq\left\|u_{n+1}\right\|_{H^{s+2}}^{2}+2 C_{0}^{2} E_{1}^{4} \nu^{-1} \\
\partial_{t}\left\|h_{n+1}\right\|_{H^{s+2}}^{2} & \leq \frac{\nu E_{2}^{2}}{4 E_{1}^{2}}\left\|\nabla u_{n}\right\|_{H^{s+2}}^{2}+\frac{5 C_{0}^{2} E_{1}^{2}}{\nu E_{2}^{2}}\left\|h_{n+1}\right\|_{H^{s+2}}^{2} .
\end{aligned}
$$

Integrating from 0 to $t$ yields

$$
\begin{gathered}
\left\|u_{n+1}(t)\right\|_{H^{s+2}}^{2}+\nu \int_{0}^{t} e^{t-\tau}\left\|\nabla u_{n+1}(\tau)\right\|_{H^{s+2}}^{2} d \tau \leq \\
\leq\left\|u_{n+1}(0)\right\|_{H^{s+2}}^{2} e^{t}+t e^{t} 2 C_{0}^{2} E_{1}^{4} \nu^{-1}, \\
\left\|h_{n+1}(t)\right\|_{H^{s+2}}^{2} \leq e^{t 5 C_{0}^{2} E_{1}^{2} \nu^{-1} E_{2}^{-2}}\left(\left\|h_{n+1}(0)\right\|_{H^{s+2}}^{2}+\frac{\nu E_{2}^{2}}{4 E_{1}^{2}} \int_{0}^{t}\left\|\nabla u_{n}\left(t^{\prime}\right)\right\|_{H^{s+2}}^{2} d t^{\prime}\right) .
\end{gathered}
$$

By the definition of $\left.\left(u_{n+1}, h_{n+1}\right)\right|_{t=0}$ we know that

$$
\begin{aligned}
\left\|u_{n+1}(0)\right\|_{H^{s+2}} & \leq\left\|u_{0}\right\|_{H^{s+2}}, \\
\left\|h_{n+1}(0)\right\|_{H^{s+2}} & \leq\left\|h_{0}\right\|_{H^{s+2}} .
\end{aligned}
$$

Thus, the choice of $T_{1}$ gives that

$$
\begin{aligned}
\left\|u_{n+1}(t)\right\|_{L^{\infty}\left(\left[0, T_{1}\right], H^{s+2}\right)}^{2}+\nu\left\|\nabla u_{n+1}(\tau)\right\|_{L^{2}\left(\left[0, T_{1}\right], H^{s+2}\right)}^{2} & \leq E_{1}^{2} \\
\left\|h_{n+1}(t)\right\|_{L^{\infty}\left(\left[0, T_{1}\right], H^{s+2}\right)}^{2} & \leq E_{2}^{2} .
\end{aligned}
$$

We have proved proposition 3.1. 


\section{Convergence for small norm}

Proposition 3.2 Let $\left(u_{0}(x), h_{0}(x)\right) \in H^{s+2}\left(\mathbb{R}^{2}\right)$ for $s>0$ and $\left\|h_{0}\right\|_{H^{s+2}} \leq$ $\frac{1}{4 C_{s}}$, then there exists a positive time $T_{2}\left(\leq T_{1}\right)$ which independent of $n$, such that $\left\{\left(u_{n}(x, t), h_{n}(x, t)\right)\right\}$ is a Cauchy sequence in $\mathcal{X}\left(\left[0, T_{2}\right], s+1, E_{1}, E_{2}\right)$ if $s \neq 1$, and in $\mathcal{X}\left(\left[0, T_{2}\right], 2-\varepsilon, E_{1}, E_{2}\right)$ for all $1>\varepsilon>0$ if $s=1$.

Proof. From equations (3.4) and (3.5), we have

$$
\begin{aligned}
& \partial_{t}\left(u_{n+1}-u_{n}\right)-\nu \triangle\left(u_{n+1}-u_{n}\right)=\sum_{j=1}^{6} F_{j}, \\
& \partial_{t}\left(h_{n+1}-h_{n}\right)+u_{n} \nabla\left(h_{n+1}-h_{n}\right)=\sum_{j=1}^{3} J_{j},
\end{aligned}
$$

where

$$
\begin{aligned}
\sum_{j=1}^{6} F_{j}= & \frac{1}{1+h_{n}} \nabla h_{n} \nabla\left(u_{n}-u_{n-1}\right) \\
& +\frac{1}{1+h_{n}} \nabla\left(h_{n}-h_{n-1}\right) \nabla u_{n-1}+\left(\frac{1}{1+h_{n}}-\frac{1}{1+h_{n-1}}\right) \nabla h_{n-1} \nabla u_{n-1} \\
& -u_{n} \nabla\left(u_{n}-u_{n-1}\right)-\left(u_{n}-u_{n-1}\right) \nabla u_{n-1}+\nabla\left(h_{n}-h_{n-1}\right), \\
\sum_{j=1}^{3} J_{j} & =\left(u_{n}-u_{n-1}\right) \nabla h_{n}+\left(1+h_{n}\right) \operatorname{div}\left(u_{n}-u_{n-1}\right)+\left(h_{n}-h_{n-1}\right) \operatorname{div} u_{n-1} .
\end{aligned}
$$

As in the proof of Proposition 3.1, applying the operator $\Delta_{k}$ to the equations (3.9) and (3.10), multiplying the first by $\Delta_{k}\left(u_{n+1}-u_{n}\right)$, and the second by $\Delta_{k}\left(h_{n+1}-h_{n}\right)$, then integrating over $\mathbb{R}^{2}$, we obtain

$$
\begin{aligned}
& \partial_{t}\left\|\Delta_{k}\left(u_{n+1}-u_{n}\right)\right\|_{L^{2}}^{2}+2 \nu\left\|\Delta_{k}\left(u_{n+1}-u_{n}\right)\right\|_{L^{2}}^{2}=\sum_{j=1}^{6} \int_{\mathbb{R}^{2}} \Delta_{k} F_{j} \Delta_{k}\left(u_{n+1}-u_{n}\right) d x, \\
& \partial_{t}\left\|\Delta_{k}\left(h_{n+1}-h_{n}\right)\right\|_{L^{2}}^{2}=\sum_{j=1}^{3} \int_{\mathbb{R}^{2}} \Delta_{k} J_{j} \Delta_{k}\left(h_{n+1}-h_{n}\right) d x
\end{aligned}
$$

Below we only consider the case of $0<s<1$. Using Lemma 2.1, Lemma 2.2 and Lemma 2.3, and the fact that $\left\|u_{n}(t)\right\|_{H^{s+1}} \leq E_{1}$ and $\left\|h_{n}(t)\right\|_{H^{s+1}} \leq E_{2}$ when $t \leq T_{1}$, we have that

$$
\begin{aligned}
\partial_{t}\left\|u_{n+1}-u_{n}\right\|_{H^{s+1}}^{2}+ & 2 \nu\left\|\nabla\left(u_{n+1}-u_{n}\right)\right\|_{H^{s+1}}^{2} \\
\leq A_{0} & \left(\left\|u_{n}-u_{n-1}\right\|_{H^{s+1}}+\left\|h_{n}-h_{n-1}\right\|_{H^{s+1}}\right) \\
& \times\left(\left\|u_{n+1}-u_{n}\right\|_{H^{s+1}}+\left\|\nabla\left(u_{n+1}-u_{n}\right)\right\|_{H^{s+1}}\right),
\end{aligned}
$$


and

$$
\begin{aligned}
\partial_{t} \| h_{n+1}- & h_{n} \|_{H^{s+1}}^{2} \leq A_{0}\left(\left\|h_{n+1}-h_{n}\right\|_{H^{s+1}}^{2}+\left(\left\|u_{n}-u_{n-1}\right\|_{H^{s+1}}\right.\right. \\
& \left.\left.+\left\|\nabla\left(u_{n}-u_{n-1}\right)\right\|_{H^{s+1}}+\left\|h_{n}-h_{n-1}\right\|_{H^{s+1}}\right)\left\|h_{n+1}-h_{n}\right\|_{H^{s+1}}\right),
\end{aligned}
$$

where $A_{0}$ is a constant, and $A_{0}=O\left(E_{1}^{4} E_{2}^{-2}\right)$. Using Cauchy-Schwarz inequality, we obtain

$$
\begin{aligned}
& \partial_{t}\left\|u_{n+1}-u_{n}\right\|_{H^{s+1}}^{2}+\nu\left\|\nabla\left(u_{n+1}-u_{n}\right)\right\|_{H^{s+1}}^{2} \\
& \quad \leq\left\|u_{n+1}-u_{n}\right\|_{H^{s+1}}^{2}+\frac{A_{0}^{2}}{\nu}\left(\left\|u_{n}-u_{n-1}\right\|_{H^{s+1}}^{2}+\left\|h_{n}-h_{n-1}\right\|_{H^{s+1}}^{2}\right),
\end{aligned}
$$

$$
\begin{aligned}
\partial_{t}\left\|h_{n+1}-h_{n}\right\|_{H^{s+1}}^{2} \leq & \widetilde{A}_{0}^{2}\left\|h_{n+1}-h_{n}\right\|_{H^{s+1}}^{2}+\frac{E_{2}^{2}}{4 E_{1}^{2}}\left\|u_{n}-u_{n-1}\right\|_{H^{s+1}}^{2} \\
& +\frac{\nu E_{2}^{2}}{4 E_{1}^{2}}\left\|\nabla\left(u_{n}-u_{n-1}\right)\right\|_{H^{s+1}}^{2}+\left\|h_{n}-h_{n-1}\right\|_{H^{s+1}}^{2},
\end{aligned}
$$

where $\widetilde{A}_{0}^{2}=4 A_{0}^{2}\left(1+\frac{E_{1}^{2}}{\nu E_{2}^{2}}\right)$.

We prove now that there exists a positive time $T_{2}\left(\leq T_{1}\right)$ such that, for any $n$,

$\left(C_{n}\right)\left\{\begin{array}{l}\left\|u_{n}-u_{n-1}\right\|_{L^{\infty}\left(\left[0, T_{2}\right], H^{s+1}\right)}+\nu\left\|\nabla\left(u_{n}-u_{n-1}\right)\right\|_{L^{2}\left(\left[0, T_{2}\right], H^{s+1}\right)} \leq E_{1} 2^{-n}, \\ \left\|h_{n}-h_{n-1}\right\|_{L^{\infty}\left(\left[0, T_{2}\right], H^{s+1}\right)} \leq E_{2} 2^{-n} .\end{array}\right.$

We will prove $\left(C_{n}\right)$ by induction on $n$. In fact, it is easy to see that $\left(C_{1}\right)$ is valid if $T_{2} \leq T_{1}$.

We suppose now that $\left(C_{n}\right)$ holds and prove that $\left(C_{n+1}\right)$ is valid using the estimates (3.11) and (3.12). Taking integration from 0 to $t$ on (3.11), we deduce

$$
\begin{aligned}
\left\|\left(u_{n+1}-u_{n}\right)(t)\right\|_{H^{s+1}}^{2} & +\nu \int_{0}^{t} e^{t-t^{\prime}}\left\|\nabla\left(u_{n+1}-u_{n}\right)\left(t^{\prime}\right)\right\|_{H^{s+1}}^{2} d t^{\prime} \\
& \leq e^{t}\left\|\left(u_{n+1}-u_{n}\right)(0)\right\|_{H^{s+1}}^{2}+t e^{t} \frac{A_{0}^{2}}{\nu}\left(E_{1}^{2}+E_{2}^{2}\right) 2^{-2 n} .
\end{aligned}
$$

If $T_{2}=\min \left\{T_{1}, \nu\left(6 A_{0}^{2}\right)^{-1}\right\}$ and $t \leq T_{2}$, we have $e^{t} \leq 3 / 2, t e^{t} 2 \frac{A_{0}^{2}}{\nu} \leq 3 / 2$, we also have

$$
\left\|\left(u_{n+1}-u_{n}\right)(0)\right\|_{H^{s+1}} \leq 2^{-(n+1)}\left\|\Delta_{n+1} u_{0}\right\|_{H^{s+2}} \leq \frac{1}{2} E_{1} 2^{-(n+1)} .
$$

Using $\left(C_{n}\right)$, we obtain

(3.13) $\left\|\left(u_{n+1}-u_{n}\right)\right\|_{L^{\infty}\left(\left[0, T_{2}\right], H^{s+1}\right)}^{2}+\nu\left\|\nabla\left(u_{n+1}-u_{n}\right)\right\|_{L^{2}\left(\left[0, t_{2}\right], H^{s+1}\right)}^{2} \leq E_{1}^{2} 2^{-2(n+1)}$. 
The same calculus for (3.12) yields

$$
\left\|\left(h_{n+1}-h_{n}\right)(t)\right\|_{H^{s+1}}^{2} \leq e^{\widetilde{A}_{0}^{2} t}\left\|\left(h_{n+1}-h_{n}\right)(0)\right\|_{H^{s+1}}^{2}+2 t e^{\widetilde{A}_{0}^{2} t} E_{2}^{2} 2^{-2 n} .
$$

Finally if $T_{2}=\min \left\{T_{1}, \nu\left(6 A_{0}^{2}\right)^{-1}, \widetilde{A}_{0}^{-2}\right\}$ and $t \leq T_{2}$, we obtain

$$
\left\|\left(h_{n+1}-h_{n}\right)\right\|_{L^{\infty}\left(\left[0, T_{2}\right], H^{s+1}\right)}^{2} \leq E_{2}^{2} 2^{-2(n+1)} .
$$

Proposition 3.2 is proved now with $T_{2}=O\left(E_{1}^{-10} \nu^{2} E_{2}^{6}\right)$.

\section{Regularity and uniqueness of solutions}

From Proposition 3.2, we may conclude that the approximative sequence $\left(u_{n}, h_{n}\right)$ of problems (3.4)-(3.6) is a Cauchy sequence in $\mathcal{X}\left(\left[0, T_{2}\right], s+1, E_{1}, E_{2}\right)$ with $s>0$. So that the limit $(u, h)$ is a solution of Cauchy problem (1.1)-(1.3). From Proposition 3.1, this sequence is bounded in $\mathcal{X}\left(\left[0, T_{1}\right], s+2, E_{1}, E_{2}\right)$, so that it is also the Cauchy sequence in $\mathcal{X}\left(\left[0, T_{2}\right], s^{\prime}+2, E_{1}, E_{2}\right)$ for all $s^{\prime}<s$ (by interpolation), and the limit is in $\mathcal{X}\left(\left[0, T_{2}\right], s+2, E_{1}, E_{2}\right)$. So we have proved the existence of solution for Theorem 3.1.

The proof of uniqueness of solution is similar to the proofs for the convergence of approximative sequence. In fact, we consider

$$
\begin{aligned}
& \partial_{t}(u-v)-\nu \triangle(u-v)=G_{1}(u, h)-G_{1}(v, g) \\
& \partial_{t}(h-g)-u \nabla(h-g)=(u-v) \nabla g+G_{2}(u, h)-G_{2}(v, g)
\end{aligned}
$$

with initial data $u(x, 0)=v(x, 0)=u_{0}(x) \in H^{s+2}$ and $h(x, 0)=g(x, 0)=$ $h_{0}(x) \in H^{s+2}$.

Following the proof of Proposition 3.2, we obtain that

$$
\begin{aligned}
\|(u-v)\|_{L^{\infty}\left(\left[0, T_{2}\right], H^{s+1}\right)}^{2}+ & \nu\|\nabla(u-v)\|_{L^{2}\left(\left[0, T_{2}\right], H^{s+1}\right)}^{2} \\
\leq & 2\|(u-v)(0)\|_{H^{s+1}}^{2}+\frac{1}{16}\left(\|u-v\|_{L^{\infty}\left(\left[0, T_{2}\right], H^{s+1}\right)}^{2}\right. \\
& \left.+\|h-g\|_{L^{\infty}\left(\left[0, T_{2}\right], H^{s+1}\right)}^{2}+\nu\|u-v\|_{L^{2}\left(\left[0, T_{2}\right], H^{s+1}\right)}^{2}\right),
\end{aligned}
$$

and

$$
\|(g-h)(t)\|_{H^{s+1}}^{2} \leq 2\|(h-g)(0)\|_{H^{s+1}}^{2}+\frac{1}{16}\left(\|u-v\|_{L^{\infty}\left(\left[0, T_{2}\right], H^{s+1}\right.}^{2}\right)
$$

$$
\left.+\|h-g\|_{L^{\infty}\left(\left[0, T_{2}\right], H^{s+1}\right)}^{2}+\nu\|u-v\|_{L^{2}\left(\left[0, T_{2}\right], H^{s+1}\right)}^{2}\right) .
$$

This gives the uniqueness of the solutions. 


\section{Global existence for small initial data}

First, we prove a priori estimates for local solutions.

Theorem 4.1 (a priori estimate) Suppose that the problem (1.1)-(1.3) has a solution $(u, h) \in L^{\infty}\left([0, T], H^{s+1}\right), \nabla u \in L^{2}\left([0, T], H^{s+1}\left(\mathbb{R}^{2}\right)\right)$ for some $T>0$ with initial data $u_{0}, h_{0} \in H^{s+1}\left(\mathbb{R}^{2}\right), s>0$, and

$$
N(T)=\left(\|u\|_{L^{\infty}\left([0, T] ; H^{s+1}\right)}^{2}+\|h\|_{L^{\infty}\left([0, T] ; H^{s+1}\right)}^{2}+\nu\|\nabla u\|_{L^{2}\left([0, T] ; H^{s+1}\right)}^{2}\right)^{1 / 2} \leq E_{0} .
$$

Then there exist positive constants $\varepsilon$ and $C_{1}$ with $\varepsilon C_{1} \leq E_{0}$, which are independent of $T$ such that, if $N(T) \leq \varepsilon$, then

$$
N(T) \leq C_{1} N(0)
$$

A combination of local existence theorem 3.1 and above a priori estimate give the following theorem.

Theorem 4.2 Suppose that $u_{0}, h_{0} \in H^{s+2}\left(\mathbb{R}^{2}\right), s>0$. Then there exists $\varepsilon>0$ such that if

$$
\left\|u_{0}\right\|_{H^{s+2}}+\left\|h_{0}\right\|_{H^{s+2}} \leq \varepsilon
$$

then the Cauchy problem (3.1)-(3.3) has a unique global solution

$$
(u, h) \in L^{\infty}\left(\left[0,+\infty\left[, H^{s+2}\left(\mathbb{R}^{2}\right)\right), \quad \nabla u \in L^{2}\left(\left[0, \infty\left[, H^{s+2}\left(\mathbb{R}^{2}\right)\right) .\right.\right.\right.\right.
$$

For the proof of this theorem we refer to Sundbye [11].

Remark. We get global solution with index $s+2$, since we have only local solution with index $s+2$ in Theorem 3.1. But we have proved the a priori estimate for small index $s+1$, so if we can get local solution for $s+1$, we will also get global solution for small index $s+1$.

We prove now Theorem 4.1. Let us linearize the equations (1.1) and (1.2) on $(h, u)=(1,0)$ as follows:

$$
\left\{\begin{array}{l}
u_{t}-\nu \triangle u+\nabla h=H_{1} \\
h_{t}+\operatorname{div} u=H_{2}
\end{array}\right.
$$

where

$$
\left\{\begin{array}{l}
H_{1}=\frac{1}{1+h} \nabla h \nabla u-(u \cdot \nabla) u, \\
H_{2}=-\operatorname{div}(h u),
\end{array}\right.
$$

In the following we will estimate $(u, h)$ under the a priori assumption

$$
\widetilde{N}(T)=\|h\|_{L^{\infty}\left([0, T], H^{s+1}\right)}^{2}+\|u\|_{L^{\infty}\left([0, T], H^{s+1}\right)}^{2} \leq \delta_{0},
$$

where $s>0$ and $0<\delta_{0}<<1$. 
Applying the operator $\Delta_{k}$ on (4.2), multiplying the first equation of (4.2) by $\Delta_{k}(u-\triangle u+\lambda \nabla h)$ and the second equation by $\Delta_{k}(h-\triangle h)$, summing them and integrating over $\mathbb{R}^{2}$ yields

$$
\begin{aligned}
\frac{1}{2} \partial_{t}\left(\left\|u_{k}\right\|_{H^{1}}^{2}+\left\|h_{k}\right\|_{H^{1}}^{2}\right)+ & \nu\left\|\nabla u_{k}\right\|_{L^{2}}^{2}+\nu\left\|\Delta u_{k}\right\|_{L^{2}}^{2}+\lambda\left\|\nabla h_{k}\right\|_{L^{2}}^{2} \\
= & \int_{\mathbb{R}^{2}}\left(\Delta_{k} F_{1} \Delta_{k}(u-\triangle u+\lambda \nabla h)+\Delta_{k} F_{2} \Delta_{k}(h-\triangle h)\right) d x \\
& \quad-\lambda \int_{\mathbb{R}^{2}}\left(\partial_{t} u_{k} \nabla h_{k}-\nu \triangle u_{k} \nabla h_{k}\right) d x,
\end{aligned}
$$

where $0<\lambda<<1, u_{k}=\triangle_{k} u, h_{k}=\triangle_{k} h$.

\section{High vertical frequencies estimates}

Now we will give some estimates to the right hand of (4.4) for the case of high vertical frequencies. This means that for some $M$ large enough, we study (4.4) for $k>M$. By lemma 2.4 we have

$$
\begin{aligned}
& \left|\int_{\mathbb{R}^{2}} \Delta_{k}\left(\frac{1}{1+h} \nabla h \nabla u\right) u_{k} d x\right| \\
& \leq C_{0} d_{k}^{2} 2^{-2 k s}\|u\|_{H^{s}}\left(1+\|h\|_{H^{s+1}}\right)\left(\|\triangle u\|_{H^{s}}^{2}+\|\nabla h\|_{H^{s}}^{2}\right),
\end{aligned}
$$

$$
\left|\int_{\mathbb{R}^{2}} \Delta_{k}\left(\frac{1}{1+h} \nabla h \nabla u\right) \triangle u_{k} d x\right| \leq C_{0} d_{k}^{2} 2^{-2 k s}\|\nabla h\|_{H^{s}}\left(1+\|h\|_{H^{s+1}}\right)\|\Delta u\|_{H^{s}}^{2}
$$

and

$$
\begin{aligned}
\lambda \mid \int_{\mathbb{R}^{2}} & \Delta_{k}\left(\frac{1}{1+h} \nabla h \nabla u\right) \nabla h_{k} d x \mid \\
& \leq C_{0} \lambda d_{k}^{2} 2^{-2 k s}\|\nabla h\|_{H^{s}}\left(1+\|h\|_{H^{s+1}}\right)\left(\|\triangle u\|_{H^{s}}^{2}+\|\nabla h\|_{H^{s}}^{2}\right) .
\end{aligned}
$$

We also have

$$
\begin{aligned}
& \left|\int_{\mathbb{R}^{2}} \Delta_{k}((u \cdot \nabla) u) \Delta u_{k} d x\right|=\left|\int_{\mathbb{R}^{2}} \Delta_{k}\left(T_{u} \nabla u+T_{\nabla u} u\right) \triangle u_{k}+R(u, \nabla u) \triangle u_{k} d x\right| \\
& \quad \leq \sum_{|q-k| \leq N_{1}}\left(\left|\int_{\mathbb{R}^{2}} \Delta_{k}\left(S_{q} u \nabla u_{q}\right) \triangle u_{k} d x\right|+\left|\int_{\mathbb{R}^{2}} \Delta_{k}\left(S_{q}(\nabla u) u_{q}\right) \triangle u_{k} d x\right|\right) \\
& \quad+\sum_{q \geq k-N_{2}, j \in\{-1,0,1\}}\left|\int_{\mathbb{R}^{2}} \Delta_{k}\left(u_{q} \nabla u_{q-j}\right) \Delta u_{k} d x\right| \\
& \quad \leq C_{0} d_{k}^{2} 2^{-2 k s}\left(\|u\|_{L^{2}}\|\triangle u\|_{H^{s}}^{2}+\|\nabla u\|_{L^{2}}\|\nabla u\|_{H^{s}}\|\triangle u\|_{H^{s}}\right) \\
& \leq C_{0} d_{k}^{2} 2^{-2 k s}\|u\|_{H^{s+1}}\|\nabla u\|_{H^{s+1}}^{2} .
\end{aligned}
$$


14 W. Wang and C-J. Xu

Similarly,

$$
\lambda\left|\int_{\mathbb{R}^{2}} \Delta_{k}((u \cdot \nabla) u) \nabla h_{k} d x\right| \leq C_{0} \lambda d_{k}^{2} 2^{-2 k s}\|u\|_{H^{s+1}}\left(\|\nabla u\|_{H^{s+1}}^{2}+\|\nabla h\|_{H^{s}}^{2}\right) .
$$

Since $\left\|f_{q}\right\|_{H^{s}} \leq\left\|\nabla f_{q}\right\|_{H^{s}}$ for $q \geq 0$, we can obtain that

$$
\left|\int_{\mathbb{R}^{2}} \Delta_{k}((u \cdot \nabla) u) u_{k} d x\right| \leq C d_{k}^{2} 2^{-2 k s}\|u\|_{H^{s}}\|\nabla u\|_{H^{s}}^{2},
$$

and

$$
\begin{aligned}
\left|\int_{\mathbb{R}^{2}} \operatorname{div} \Delta_{k}(u h) h_{k} d x\right| & =\left|\int_{\mathbb{R}^{2}} \Delta_{k}(u h) \nabla h_{k} d x\right| \\
& \leq C d_{k}^{2} 2^{-2 k s}\left(\|u\|_{L^{2}}+\|h\|_{L^{2}}\right)\left(\|\nabla u\|_{H^{s}}^{2}+\|\nabla h\|_{H^{s}}^{2}\right) .
\end{aligned}
$$

Using lemma 2.5, we obtain

$$
\mid \int_{\mathbb{R}^{2}} \Delta_{k}\left(\operatorname{div}(h u) \triangle h_{k} d x \mid \leq C d_{k}^{2} 2^{-2 k s}\|\nabla h\|_{H^{s}}\left(\|\nabla h\|_{H^{s}}^{2}+\|\nabla u\|_{H^{s+1}}^{2}\right) .\right.
$$

It is easy to see that

$$
\lambda \nu\left|\int_{\mathbb{R}^{2}} \triangle u_{k} \nabla h_{k} d x\right| \leq C \lambda \nu d_{k}^{2} 2^{-2 k s}\left(\varepsilon^{-1}\|\triangle u\|_{H^{s}}^{2}+\varepsilon\|\nabla h\|_{H^{s}}^{2}\right) .
$$

Noting that

$$
\int_{\mathbb{R}^{2}}\left(\partial_{t} u_{k}\right)\left(\nabla h_{k}\right) d x=\partial_{t} \int_{\mathbb{R}^{2}} u_{k} \nabla h_{k} d x-\int_{\mathbb{R}^{2}} u_{k} \partial_{t}\left(\nabla h_{k}\right) d x
$$

we have

$$
\begin{aligned}
\lambda\left|\int_{\mathbb{R}^{2}} u_{k} \partial_{t}\left(\nabla h_{k}\right) d x\right| \leq & C \lambda d_{k}^{2} 2^{-2 k s}\left(\|\nabla u\|_{H^{s}}^{2}\right. \\
& \left.+\left(\|h\|_{H^{s+1}}+\|u\|_{H^{s+1}}\right)\left(\|\nabla u\|_{H^{s}}^{2}+\|\nabla h\|_{H^{s}}^{2}\right)\right)
\end{aligned}
$$

and

$$
\begin{aligned}
& \lambda\left|\int_{0}^{t} \partial_{\tau}\left(\int_{\mathbb{R}^{2}} u_{k} \nabla h_{k} d x\right) d \tau\right| \leq C \lambda d_{k}^{2} 2^{-2 k s}\left(\|u(t)\|_{H^{s}}\|\nabla h(t)\|_{H^{s}}\right. \\
&\left.+\|u(0)\|_{H^{s}}\|\nabla h(0)\|_{H^{s}}\right) .
\end{aligned}
$$


Multiplying inequality (4.4) by $2^{2 k s}$ and integrating over $(0, t)$, we obtain

$$
\begin{gathered}
\left\|u_{k}(t)\right\|_{H^{s+1}}^{2}+\left\|h_{k}(t)\right\|_{H^{s+1}}^{2}+\int_{0}^{t}\left(\nu\left\|\nabla u_{k}(\tau)\right\|_{H^{s+1}}^{2}+\lambda\left\|\nabla h_{k}(\tau)\right\|_{H^{s}}^{2}\right) d \tau \\
\leq C d_{k}^{2}\left(\|u(0)\|_{H^{s+1}}^{2}+\|h(0)\|_{H^{s+1}}^{2}\right)+C \lambda \nu d_{k}^{2} \int\left(\varepsilon^{-1}\|\nabla u\|_{H^{s+1}}^{2}+\varepsilon\|\nabla h\|_{H^{s}}^{2}\right) d \tau \\
+C d_{k}^{2}\left(\|h\|_{L^{\infty}\left([0, T], H^{s+1}\right)}+\|h\|_{L^{\infty}\left([0, T], H^{s+1}\right)}^{2}\right. \\
\left.\quad+\|u\|_{L^{\infty}\left([0, T], H^{s+1}\right)}+\|u\|_{L^{\infty}\left([0, T], H^{s+1}\right)}^{2}\right) \int_{0}^{t}\left(\|\nabla u\|_{H^{s+1}}^{2}+\|\nabla h\|_{H^{s}}^{2}\right) d \tau \\
+C \lambda d_{k}^{2}\left(\|u(t)\|_{H^{s}}^{2}+\|h(t)\|_{H^{s+1}}^{2}\right) .
\end{gathered}
$$

\section{Low vertical frequencies estimates}

Now we will consider the low vertical frequencies: denoting $S_{M}=\sum_{k<M} \Delta_{k}$, applying the operator $S_{M}$ on (4.2), multiplying the first equation of (4.2) by $S_{k}(u+\lambda \nabla h)$ and the second equation by $S_{k} h$, summing them and integrating over $\mathbb{R}^{2}$ yields

$$
\begin{aligned}
\frac{1}{2} \partial_{t}\left(\left\|S_{M} u\right\|_{L^{2}}^{2}+\right. & \left.\left\|S_{M} h\right\|_{L^{2}}^{2}\right)+\nu\left\|\nabla S_{M} u\right\|_{L^{2}}^{2}+\lambda\left\|\nabla S_{M} h\right\|_{L^{2}}^{2} \\
= & \int_{\mathbb{R}^{2}}\left(S_{M}\left(F_{1}\right) S_{M}(u+\lambda \nabla h)+S_{M}\left(F_{2}\right) S_{M} h\right) d x \\
& -\lambda \int_{\mathbb{R}^{2}}\left(\partial_{t} S_{M} u \nabla S_{M} h-\nu \triangle S_{M} u \nabla S_{M} h\right) d x,
\end{aligned}
$$

where $0<\lambda<<1$. As in the proof of (4.8), we will give some estimates to the right hand of (4.9). It is easy to see that

$$
\left|\int_{\mathbb{R}^{2}} S_{M}\left(\frac{\nabla h}{1+h} \nabla u\right) S_{M} u d x\right| \leq C\left\|\frac{1}{1+h}\right\|_{L^{\infty}}\|\nabla h\|_{L^{2}}\|u\|_{L^{\infty}}\|\nabla u\|_{L^{2}}
$$

and

$$
\begin{aligned}
\lambda\left|\int_{\mathbb{R}^{2}} S_{M}\left(\frac{\nabla h}{1+h} \nabla u-(u \cdot \nabla) u\right) S_{M}(\nabla h) d x\right| \\
\leq C \lambda\left(\left\|\frac{1}{1+h}\right\|_{L^{\infty}}\|\nabla h\|_{L^{2}}^{2}\|\nabla u\|_{L^{\infty}}+\|u\|_{L^{\infty}}\|\nabla u\|_{L^{2}}\|\nabla h\|_{L^{2}}\right) .
\end{aligned}
$$

Using the estimates (2.2), we have

$$
\left|\int_{\mathbb{R}^{2}} S_{M}((u \cdot \nabla) u) S_{M} u d x\right| \leq C\|u\|_{\dot{H}^{1 / 2}}^{2}\|\nabla u\|_{L^{2}} \leq C\|u\|_{L^{2}}\|\nabla u\|_{L^{2}}^{2},
$$


16 W. WANG AND C-J. XU

and

$$
\begin{aligned}
\left|\int_{\mathbb{R}^{2}} S_{M}(\operatorname{div}(u h)) S_{M} h d x\right| & \leq C\|u\|_{\dot{H}^{1 / 2}}\|h\|_{\dot{H}^{1 / 2}}\|\nabla h\|_{L^{2}} \\
& \leq C\left(\|u\|_{L^{2}}+\|h\|_{L^{2}}\right)\left(\|\nabla u\|_{L^{2}}^{2}+\|\nabla h\|_{L^{2}}^{2}\right) .
\end{aligned}
$$

Note that $\left\|S_{M} \triangle u\right\|_{L^{2}} \leq 2^{M}\left\|S_{M} \nabla u\right\|_{L^{2}}$, which implies

$$
\left|\lambda \nu \int_{\mathbb{R}^{2}} S_{M}(\triangle u) S_{M}(\nabla h) d x\right| \leq C \lambda \nu\left(\varepsilon^{-1}\|\nabla u\|_{L^{2}}^{2}+\varepsilon\|\nabla h\|_{L^{2}}^{2}\right) .
$$

As in the above proofs, we have

$$
\begin{aligned}
\lambda\left|\int_{\mathbb{R}^{2}} S_{M} u S_{M}\left(\partial_{t}(\nabla h)\right) d x\right| \leq & C \lambda\left(\|h\|_{L^{2}}+\|u\|_{L^{2}}\right)\left(\|\nabla u\|_{L^{2}}^{2}+\|\nabla h\|_{L^{2}}^{2}\right) \\
& +C \lambda\left(\varepsilon^{-1}\|\nabla u\|_{L^{2}}^{2}+\varepsilon\|\nabla h\|_{L^{2}}^{2}\right)
\end{aligned}
$$

and

$$
\lambda\left|\int_{0}^{t} \partial_{t} \int_{\mathbb{R}^{2}} S_{M} u S_{M}(\nabla h) d x d \tau\right| \leq C \lambda\left(\|u\|_{L^{2}}\|\nabla h\|_{L^{2}}+\|u(0)\|_{L^{2}}\|\nabla h(0)\|_{L^{2}}\right) .
$$

Integrating both sides of (4.9) over $(0, t)$, and adding the above estimates to the right hand of (4.9), we have

$$
\begin{aligned}
\left(\left\|S_{M} u\right\|_{L^{2}}^{2}\right. & \left.+\left\|S_{M} h\right\|_{L^{2}}^{2}\right)+\int_{0}^{t}\left(\nu\left\|\nabla S_{M} u\right\|_{L^{2}}^{2}+\lambda\left\|\nabla S_{M} h\right\|_{L^{2}}^{2}\right) d \tau \\
\leq & C\left(\|u\|_{L^{\infty}\left([0, T], H^{s+1}\right)}+\|h\|_{L^{\infty}\left([0, T], H^{s+1}\right)}\right) \int_{0}^{t}\left(\|\nabla u\|_{H^{s}}^{2}+\|\nabla h\|_{H^{s}}^{2}\right) d \tau \\
& +C \lambda\left(\|u(t)\|_{L^{2}}^{2}+\|\nabla h(t)\|_{L^{2}}^{2}\right)+C\left(\|u(0)\|_{L^{2}}^{2}+\|\nabla h(0)\|_{L^{2}}^{2}\right) \\
& +C \lambda \nu \int_{0}^{t}\left(\varepsilon^{-1}\|\nabla u\|_{L^{2}}^{2}+\varepsilon\|\nabla h\|_{L^{2}}^{2}\right) d \tau
\end{aligned}
$$

Since $\left\|S_{M} f\right\|_{H^{s}} \leq 2^{M s}\left\|S_{M} f\right\|_{L^{2}}$, we can write

$$
\begin{aligned}
\left(\left\|S_{M} u\right\|_{H^{s+1}}^{2}\right. & \left.+\left\|S_{M} h\right\|_{H^{s+1}}^{2}\right)+\int_{0}^{t}\left(\left\|\nabla S_{M} u\right\|_{H^{s+1}}^{2}+\lambda\left\|\nabla S_{M} h\right\|_{H^{s}}^{2}\right) d \tau \\
\leq C & \left.\|u\|_{L^{\infty}\left([0, T], H^{s+1}\right)}+\|h\|_{L^{\infty}\left([0, T], H^{s+1}\right)}\right) \int_{0}^{t}\left(\|\nabla u\|_{H^{s+1}}^{2}+\|\nabla h\|_{H^{s}}^{2}\right) d \tau \\
& +C \lambda\left(\|u(t)\|_{H^{s}}^{2}+\|\nabla h(t)\|_{H^{s}}^{2}\right)+C\left(\|u(0)\|_{H^{s}}^{2}+\|\nabla h(0)\|_{H^{s}}^{2}\right) \\
& +\lambda \int_{0}^{t}\left(\varepsilon^{-1}\|\nabla u\|_{H^{s}}^{2}+\varepsilon\|\nabla h\|_{H^{s}}^{2}\right) d \tau
\end{aligned}
$$


The inequality above, together with estimate (4.8), yield:

$$
\begin{aligned}
\|u(t)\|_{H^{s+1}}^{2}+\|h(t)\|_{H^{s+1}}^{2}+\int_{0}^{t}\left(\|\nabla u(\tau)\|_{H^{s+1}}^{2}+\lambda\|\nabla h(\tau)\|_{H^{s}}^{2}\right) d \tau \\
\leq C\left(\|h\|_{L^{\infty}\left([0, T], H^{s+1}\right)}+\|u\|_{L^{\infty}\left([0, T], H^{s+1}\right)}+\|h\|_{L^{\infty}\left([0, T], H^{s+1}\right)}^{2}+\|u\|_{L^{\infty}\left([0, T], H^{s+1}\right)}^{2}\right) \\
\quad \times \int_{0}^{t}\left(\|\nabla u\|_{H^{s+1}}^{2}+\|\nabla h\|_{H^{s}}^{2}\right) d \tau+C\left(\|u(0)\|_{H^{s+1}}^{2}+\|h(0)\|_{H^{s+1}}^{2}\right) \\
\quad+C \lambda\left(\|u(t)\|_{H^{s+1}}^{2}+\|h(t)\|_{H^{s+1}}^{2}\right)+C \lambda \nu \int_{0}^{t}\left(\varepsilon^{-1}\|\nabla u\|_{H^{s+1}}^{2}+\varepsilon\|\nabla h\|_{H^{s}}^{2}\right) d \tau .
\end{aligned}
$$

Taking $\varepsilon$ and $\lambda$ small enough, such that $C \varepsilon=\frac{1}{2}$ and $C \lambda \varepsilon^{-1}=\frac{1}{4}$, we get

$$
\begin{aligned}
\| u(t) & \left\|_{H^{s+1}}^{2}+\right\| h(t) \|_{H^{s+1}}^{2}+\int_{0}^{t}\left(\nu\|\nabla u(\tau)\|_{H^{s+1}}^{2}+\lambda\|\nabla h(\tau)\|_{H^{s}}^{2}\right) d \tau \\
\leq & C\left(\|h\|_{L^{\infty}\left([0, T], H^{s+1}\right)}+\|h\|_{L^{\infty}\left([0, T], H^{s+1}\right)}^{2}+\|u\|_{L^{\infty}\left([0, T], H^{s+1}\right)}+\|u\|_{L^{\infty}\left([0, T], H^{s+1}\right)}^{2}\right) \\
& \times \int_{0}^{t}\left(\nu\|\nabla u\|_{H^{s+1}}^{2}+\lambda\|\nabla h\|_{H^{s}}^{2}\right) d \tau+C\left(\|u(0)\|_{H^{s+1}}^{2}+\|h(0)\|_{H^{s+1}}^{2}\right) .
\end{aligned}
$$

For fixed $\lambda$, taking $\delta_{0}$ small enough, such that $C \delta_{0}<\frac{1}{5} \min \{1, \lambda\}$, we obtain

$$
\begin{aligned}
&\|u(t)\|_{H^{s+1}}^{2}+\|h(t)\|_{H^{s+1}}^{2}+\int_{0}^{t}\left(\nu\|\nabla u(\tau)\|_{H^{s+1}}^{2}+\lambda\|\nabla h(\tau)\|_{H^{s}}^{2}\right) d \tau \\
& \leq C\left(\|u(0)\|_{H^{s+1}}^{2}+\|h(0)\|_{H^{s+1}}^{2}\right) .
\end{aligned}
$$

This proves Theorem 4.1.

\section{Losing energy estimates}

We prove now the losing energy estimates of section 2. The proofs of this section are technical.

Lemma 5.1 Let $\tau>1$ and $-1 \leq k<+\infty$, then there exists $C>0$ such that for all $v, \nabla v, g, \nabla g \in H^{\tau}$, we have

$$
\left|\int_{\mathbb{R}^{2}} \Delta_{k}(v \nabla g) \Delta_{k} g d x\right| \leq C d_{k}^{2} 2^{-2 k \tau}\|v\|_{H^{\tau+1}}\|g\|_{H^{\tau}}^{2}
$$

with $\left\{d_{k}\right\} \in \ell^{2}$ and $\left\|\left\{d_{k}\right\}\right\|_{\ell^{2}} \leq 1$.

Proof. Using the paraproduct calculus, we have

$$
\begin{aligned}
\int_{\mathbb{R}^{2}} \Delta_{k}(v \nabla g) \Delta_{k} g d x= & \int_{\mathbb{R}^{2}} \Delta_{k}\left(T_{\nabla g} v\right) \Delta_{k} g d x+\int_{\mathbb{R}^{2}} \Delta_{k}\left(T_{v} \nabla g\right) \Delta_{k} g d x \\
& +\int_{\mathbb{R}^{2}} \Delta_{k} R(v, \nabla g) \Delta_{k} g d x=I_{1}+I_{2}+I_{3} .
\end{aligned}
$$


Then there exists $N_{1}>0$ such that for any fixed $M>N_{1}$ and $k>M$,

$$
\begin{aligned}
\left|I_{1}\right| & \leq \sum_{|q-k| \leq N_{1}}\left\|S_{q}(\nabla g)\right\|_{L^{\infty}}\left\|\Delta_{q} v\right\|_{L^{2}}\left\|\Delta_{k} g\right\|_{L^{2}} \\
& \leq \sum_{|q-k| \leq N_{1}}\left\|S_{q} g\right\|_{L^{\infty}}\left\|\Delta_{q}(\nabla v)\right\|_{L^{2}}\left\|\Delta_{k} g\right\|_{L^{2}} \leq C d_{k}^{2} 2^{-2 k \tau}\|g\|_{H^{\tau}}^{2}\|v\|_{H^{\tau+1}} .
\end{aligned}
$$

Here we have used Sobolev inequality for $\|g\|_{L^{\infty}}$ since $\tau>1$. For $k \leq M$, using that $\sum_{|q-k| \leq N_{1}}\left\|S_{q}(\nabla g)\right\|_{L^{\infty}} \leq C 2^{M}\|g\|_{L^{\infty}}$, we get the same results.

For the term $I_{2}$, in order to pass the operator $\nabla$ from $g$ to $v$, let us rewrite

$$
\begin{aligned}
I_{2}= & \sum_{|q-k| \leq N_{1}} \int_{\mathbb{R}^{2}} \Delta_{k}\left(S_{q} v \Delta_{q}(\nabla g)\right) \Delta_{k} g d x \\
= & \sum_{|q-k| \leq N_{1}}\left(\int_{\mathbb{R}^{2}}\left[\Delta_{k}, S_{q} v\right] \Delta_{q}(\nabla g) \Delta_{k} g d x+\int_{\mathbb{R}^{2}}\left(S_{q}-S_{k}\right) v \Delta_{k} \Delta_{q}(\nabla g) \Delta_{k} g d x\right. \\
& \left.+\int_{\mathbb{R}^{2}} S_{k} v \Delta_{k}(\nabla g) \Delta_{k} g d x\right) .
\end{aligned}
$$

Note that the operators $\Delta_{k}$ are convolution operators in $\mathbb{R}^{2}$, therefore

$$
\left[\Delta_{k}, S_{q} v\right] \Delta_{q}(\nabla g)=2^{2 k} \int_{\mathbb{R}^{2}}\left(S_{q} v(x)-S_{q} v(y)\right) f\left(2^{k}(x-y)\right) \Delta_{q}(\nabla g)(y) d y,
$$

where $f(x)=\left(\mathcal{F}^{-1} \varphi\right)(x)$. Using the fact that $|q-k| \leq N_{1}$ and HausdorffYoung inequality, we have

$$
\begin{aligned}
\sum_{|q-k| \leq N_{1}}\left\|\left[\Delta_{k}, S_{q} v\right] \Delta_{q}(\nabla g)\right\|_{L^{2}} \\
\leq C \sum_{|q-k| \leq N_{1}} 2^{2 k}\left\|\nabla\left(S_{q} v\right)\right\|_{L^{\infty}} 2^{-k}\left\|\left(2^{k} \cdot\right) f\left(2^{k} \cdot\right)\right\|_{L^{1}}\left\|\Delta_{q}(\nabla g)\right\|_{L^{2}} \\
\leq C d_{k} 2^{-k \tau}\|\nabla v\|_{L^{\infty}}\|g\|_{H^{\tau}}
\end{aligned}
$$

A similar computation for other terms yields :

$$
\left|I_{2}\right| \leq C d_{k}^{2} 2^{-2 k \tau}\|\nabla v\|_{H^{\tau}}\|g\|_{H^{\tau}}^{2} .
$$

Finally, for $I_{3}$, there exists $N_{1}>0$ such that

$$
\begin{aligned}
\left|I_{3}\right| & \leq \sum_{q \geq k-N_{2}, j \in\{-1,0,1\}}\left|\int_{\mathbb{R}^{2}} \Delta_{k}\left(\Delta_{q} v \Delta_{q-j}(\nabla g)\right) \Delta_{k} g d x\right| \\
& \leq C \sum_{q \geq k-N_{2}}\left\|\Delta_{q} v\right\|_{L^{2}}\left\|\Delta_{q-j}(\nabla g)\right\|_{L^{\infty}}\left\|\Delta_{k} g\right\|_{L^{2}} \\
& \leq C d_{k} 2^{-2 k \tau}\left(\sum_{q \geq k-N_{2}} d_{q} 2^{-(q-k) \tau}\right)\|v\|_{H^{\tau+1}}\|g\|_{H^{\tau}}^{2} .
\end{aligned}
$$


Denote $d_{k}^{\prime}=\left(\sum_{q \geq k-N_{2}} d_{q} 2^{-(q-k) \tau}\right)$, then $\left\{d_{k}^{\prime}\right\} \in l^{2}$ since $q>k$ and $\tau>1$. For the sake of convenience, below we also denote $d_{k}^{\prime}$ by $d_{k}$. Thus

$$
\left|I_{3}\right| \leq C d_{k}^{2} 2^{-2 k \tau}\|v\|_{H^{\tau+1}}\|g\|_{H^{\tau}}^{2}
$$

Lemma 5.1 is proved.

Lemma 5.2 (a) Let $\tau>2$ and $-1 \leq k<+\infty$, then there exists $C>0$ such that for all $f, v, g, u, \nabla u \in H^{\tau}$, we have

$\left|\int_{\mathbb{R}^{2}} \Delta_{k}\left(\frac{\nabla f}{1+g} \nabla v\right) \Delta_{k} u d x\right| \leq C d_{k}^{2} 2^{-2 k \tau} H_{0}(g)\|f\|_{H^{\tau}}\|v\|_{H^{\tau}}\left(1+\|g\|_{H^{\tau}}\right)\|u\|_{H^{\tau+1}}$, with $\left\{d_{k}\right\} \in \ell^{2}$, where

$$
H_{0}(g)=1+\left\|(1+g)^{-1}\right\|_{L^{\infty}}+B_{0}\left(\|g\|_{L^{\infty}}\right),
$$

and $B_{0}$ is the function give in (2.1).

(b) Let $1<\tau<2$ and $-1 \leq k<+\infty$, then there exists $C>0$ such that for all $f, g, u, \nabla u, v, \nabla v \in H^{\tau}$, we have

$$
\left|\int_{\mathbb{R}^{2}} \Delta_{k}\left(\frac{\nabla f}{1+g} \nabla v\right) \Delta_{k} u d x\right| \leq C d_{k}^{2} 2^{-2 k \tau} H_{0}(g)\|f\|_{H^{\tau}}\left(1+\|g\|_{H^{\tau}}\right) U_{\tau}(u, v),
$$

with $\left\{d_{k}\right\} \in \ell^{2}$, where

$$
U_{\tau}(u, v)=:\|\nabla v\|_{L^{\infty}}\|u\|_{H^{\tau+1}}+\|\nabla v\|_{H^{\tau}}\left(\|\nabla u\|_{H^{1}}+\|u\|_{H^{\tau}}\right) .
$$

Proof. (a) As in the proof of Lemma 5.1, first we have

$$
\int_{\mathbb{R}^{2}} \Delta_{k}\left(\frac{\nabla f}{1+g} \nabla v\right) \Delta_{k} u d x=\int_{\mathbb{R}^{2}} \Delta_{k}\left(T_{\frac{\nabla f}{1+g}} \nabla v+T_{\nabla v} \frac{\nabla f}{1+g}+R\left(\frac{\nabla f}{1+g}, \nabla v\right)\right) \Delta_{k} u d x .
$$

For $k>M$, it is easy to obtain

$$
\begin{aligned}
\left|\int_{\mathbb{R}^{2}} \Delta_{k}\left(T_{\frac{\nabla f}{1+g}} \nabla v\right) \Delta_{k} u d x\right| & \leq \sum_{|q-k| \leq N_{1}}\left\|\frac{\nabla f}{1+g}\right\|_{L^{\infty}}\left\|\Delta_{q}(\nabla v)\right\|_{L^{2}}\left\|\Delta_{k} u\right\|_{L^{2}} \\
& \leq \sum|q-k| \leq N_{1}\left\|\frac{\nabla f}{1+g}\right\|_{L^{\infty}} 2^{q}\left\|\Delta_{q} v\right\|_{L^{2}} 2^{-k}\left\|\Delta_{k} \nabla u\right\|_{L^{2}} \\
& \leq C d_{k}^{2} 2^{-2 k \tau}\left\|\frac{1}{1+g}\right\|_{L^{\infty}}\|f\|_{H^{\tau}}\|v\|_{H^{\tau}}\|\nabla u\|_{H^{\tau}} .
\end{aligned}
$$

For $k \leq M$, we have

$$
\left|\int_{\mathbb{R}^{2}} \Delta_{k}\left(T_{\frac{\nabla f}{1+g}} \nabla u\right) \Delta_{k} u d x\right| \leq C d_{k}^{2} 2^{-2 k \tau}\left\|\frac{1}{1+g}\right\|_{L^{\infty}}\|f\|_{H^{\tau}}\|v\|_{H^{\tau}}\|u\|_{H^{\tau}} .
$$


For the second term, we rewrite

$$
\Delta_{q}\left(\frac{\nabla f}{1+g}\right)=\Delta_{q}(\nabla f)-\Delta_{q}\left(T_{\frac{g}{1+g}} \nabla f+T_{\nabla f} \frac{g}{1+g}+R\left(\nabla f, \frac{g}{1+g}\right)\right),
$$

thus we have

$$
\begin{aligned}
\mid \int_{\mathbb{R}^{2}} \Delta_{k} & \left(T_{\nabla v} \frac{\nabla f}{1+g}\right) \Delta_{k} u d x \mid \\
& \leq C d_{k}^{2} 2^{-2 k \tau} H_{0}(g)\|\nabla v\|_{H^{\tau}}\|f\|_{H^{\tau}}\left(1+\|g\|_{H^{\tau}}\right)\left(\|\nabla u\|_{H^{\tau}}+\|u\|_{H^{\tau}}\right),
\end{aligned}
$$

and

$$
\begin{aligned}
& \mid \int_{\mathbb{R}^{2}} \Delta_{k}\left(R\left(\nabla v, \frac{\nabla f}{1+g}\right) \Delta_{k} u d x \mid\right. \\
& \leq \sum_{q \geq k-N_{2}, j \in\{-1,0,1\}}\left|\int_{\mathbb{R}^{2}} \Delta_{k}\left(\Delta_{q}\left(\frac{\nabla f}{1+g}\right) \Delta_{q-j}(\nabla v)\right) \Delta_{k} u d x\right| \\
& \leq C d_{k}^{2} 2^{-2 k \tau} H_{0}(g)\|\nabla v\|_{H^{\tau}}\|f\|_{H^{\tau}}\|u\|_{H^{\tau+1}}
\end{aligned}
$$

Therefore, part (a) is proved.

(b) Let us write

$$
\begin{aligned}
& \mid \int_{\mathbb{R}^{2}} \Delta_{k}(\left.\frac{\nabla f}{1+g} \nabla v\right) \Delta_{k} u d x|\leq| \int_{\mathbb{R}^{2}} \Delta_{k}\left(T_{\frac{\nabla v}{1+g}} \nabla f\right) \Delta_{k} u d x \mid \\
& \quad+\left|\int_{\mathbb{R}^{2}} \Delta_{k}\left(T_{\nabla f} \frac{\nabla v}{1+g}\right) \Delta_{k} u d x\right|+\left|\int_{\mathbb{R}^{2}} \Delta_{k} R\left(\frac{\nabla v}{1+g}, \nabla f\right) \Delta_{k} u d x\right| .
\end{aligned}
$$

The estimates for the first term and the third term are easy, so we discuss only the second term for which we consider two cases:

1) $k>M$. We have that

$$
\left|\int_{\mathbb{R}^{2}} \Delta_{k}\left(T_{\nabla f} \frac{\nabla v}{1+g}\right) \Delta_{k} u d x\right| \leq \sum_{|q-k| \leq N_{1}}\left\|S_{q}(\nabla f)\right\|_{L^{\infty}}\left\|\Delta_{q}\left(\frac{\nabla v}{1+g}\right)\right\|_{L^{2}}\left\|\Delta_{k} u\right\|_{L^{2}} .
$$

Since $1<\tau<2$, using the Sobolev's inequality, we obtain

$$
\left\|S_{q}(\nabla f)\right\|_{L^{\infty}} \leq \sum_{p \leq q+2} 2^{2 p}\left\|\Delta_{p} f\right\|_{L^{2}} \leq C 2^{-q(\tau-2)}\|f\|_{H^{\tau}}
$$

and

$\left\|\frac{\nabla v}{1+g}\right\|_{H^{\tau}} \leq\|\nabla v\|_{H^{\tau}}\left(1+\left\|\frac{g}{1+g}\right\|_{H^{\tau}}\right) \leq\|\nabla v\|_{H^{\tau}}\left(1+\left\|\left(\frac{1}{1+g}\right)^{2}\right\|_{L^{\infty}}\|g\|_{H^{\tau}}\right)$, 
which implies

$\left|\int_{\mathbb{R}^{2}} \Delta_{k}\left(T_{\nabla f} \frac{\nabla v}{1+g}\right) \Delta_{k} u d x\right| \leq C d_{k}^{2} 2^{-2 k \tau} H_{0}(g)\left(1+\|g\|_{H^{\tau}}\right)\|f\|_{H^{\tau}}\|\nabla v\|_{H^{\tau}}\|\nabla u\|_{H^{1}}$.

2) $k \leq M$. It is easy to see that

$$
\left|\int_{\mathbb{R}^{2}} \Delta_{k}\left(T_{\nabla f} \frac{\nabla v}{1+g}\right) \Delta_{k} u d x\right| \leq C d_{k}^{2} 2^{-2 k \tau} H_{0}(g)\left(1+\|g\|_{H^{\tau}}\right)\|f\|_{H^{\tau}}\|\nabla v\|_{H^{\tau}}\|u\|_{H^{\tau}} .
$$

And the lemma is proved.

Lemma 5.3 (a) Let $\tau>2$ and $-1 \leq k<+\infty$, then there exists $C>0$ such that for all $f, v, u, \nabla u, g_{1}, g_{2} \in H^{\tau}$, we have

$$
\begin{aligned}
\mid \int_{\mathbb{R}^{2}} \Delta_{k} & \left(\frac{\left(g_{1}-g_{2}\right)}{\left(1+g_{1}\right)\left(1+g_{2}\right)} \nabla f \nabla v\right) \Delta_{k} u d x \mid \\
& \leq C d_{k}^{2} 2^{-2 k \tau} H_{1}\left(g_{1}, g_{2}\right)\|f\|_{H^{\tau}}\|v\|_{H^{\tau}}\left\|g_{1}-g_{2}\right\|_{H^{\tau}}\left(\|\nabla u\|_{H^{\tau}}+\|u\|_{H^{\tau}}\right),
\end{aligned}
$$

with $\left\{d_{k}\right\} \in \ell^{2}$, and

$$
\begin{aligned}
H_{1}\left(g_{1}, g_{2}\right)= & \left(1+\left\|\left(1+g_{1}\right)^{-1}\right\|_{L^{\infty}}^{2}\left\|g_{1}\right\|_{H^{\tau}}\right)\left(1+\left\|\left(1+g_{2}\right)^{-1}\right\|_{L^{\infty}}^{2}\left\|g_{2}\right\|_{H^{\tau}}\right) \\
& +\left\|\left(1+g_{1}\right)^{-1}\right\|_{L^{\infty}}^{2}\left\|\left(1+g_{2}\right)^{-1}\right\|_{L^{\infty}}^{2} .
\end{aligned}
$$

(b) Let $1<\tau<2$ and $-1 \leq k<+\infty$, then there exists $C>0$ such that for all $f, v, g_{1}, g_{2}, u, \nabla u, v, \nabla v \in H^{\tau}$, we have

$$
\begin{aligned}
& \mid \int_{\mathbb{R}^{2}} \Delta_{k}\left(\frac{\left(g_{1}-g_{2}\right)}{\left(1+g_{1}\right)\left(1+g_{2}\right)}\right.\nabla f \nabla v) \Delta_{k} u d x \mid \\
& \leq C d_{k}^{2} 2^{-2 k \tau} H_{1}\left(g_{1}, g_{2}\right)\|f\|_{H^{\tau}}\left\|g_{1}-g_{2}\right\|_{H^{\tau}} U_{\tau}(u, v),
\end{aligned}
$$

with $\left\{d_{k}\right\} \in \ell^{2}$, and $U_{\tau}(u, v)$ as in Lemma 5.2 (b).

The proof of this lemma is similar to Lemma 5.2. Let us remark that if $F_{j}=\frac{1}{1+g_{j}}, \bar{F}_{j}=\frac{g_{j}}{1+g_{j}}(j=1,2)$, then we have

$$
F=\frac{g_{1}-g_{2}}{\left(1+g_{1}\right)\left(1+g_{2}\right)}=\left(g_{1}-g_{2}\right) F_{1} F_{2}=\left(g_{1}-g_{2}\right)\left(1-\bar{F}_{1}-\bar{F}_{2}+\bar{F}_{1} \bar{F}_{2}\right)
$$

with the following estimates

$$
\begin{aligned}
\|F\|_{L^{\infty}} & \leq C\left\|g_{1}-g_{2}\right\|_{L^{\infty}}\left\|F_{1}\right\|_{L^{\infty}}\left\|F_{2}\right\|_{L^{\infty}}, \\
\left\|\Delta_{q} F\right\|_{L^{2}} & \leq C d_{q}^{2} 2^{-2 q \tau}\left\|g_{1}-g_{2}\right\|_{H^{\tau}}\left(1+\left\|F_{1}\right\|_{L^{\infty}}^{2}\left\|g_{1}\right\|_{H^{\tau}}\right)\left(1+\left\|F_{2}\right\|_{L^{\infty}}^{2}\left\|g_{2}\right\|_{H^{\tau}}\right) .
\end{aligned}
$$

In the following we will consider the losing energy estimate for the case of high vertical frequencies, i. e., $k>M$. Here, we assume that $M>N_{1}+N_{2}$. 
Lemma 5.4 Let $\tau>0$ and $M \leq k<\infty$, then there exists $C>0$ such that for all $g, u, v, \nabla g, \nabla u \in H^{\tau}$, we have

$$
\begin{aligned}
\mid \int_{\mathbb{R}^{2}} \Delta_{k}\left(\frac{1}{1+h}\right. & \nabla h \nabla u) \Delta_{k} v d x \mid \\
& \leq C d_{k}^{2} 2^{-2 k \tau} H_{0}(h)\left(1+\|h\|_{H^{\tau+1}}\right)\|\triangle u\|_{H^{\tau}}\|\nabla h\|_{H^{\tau}}\|v\|_{H^{\tau}},
\end{aligned}
$$

with $\left\{d_{k}\right\} \in l^{2}$.

The proof of this lemma is similar to the proof of lemma 5.2 and the following lemma.

Lemma 5.5 Let $\tau>0$ and $M \leq k<\infty$, then there exists $C>0$ such that for all $g \in H^{\tau+1}$ and $u \in H^{\tau+2}$, we have

$$
\left|\int_{\mathbb{R}^{2}} \Delta_{k}(\operatorname{div}(h u)) \Delta\left(\Delta_{k} h\right) d x\right| \leq C d_{k}^{2} 2^{-2 k \tau}\|\nabla h\|_{H^{\tau}}\left(\|\nabla h\|_{H^{\tau}}^{2}+\|\nabla u\|_{H^{\tau+1}}^{2}\right),
$$

with $\left\{d_{k}\right\} \in l^{2}$.

Proof. First, let us observe that

$$
\begin{aligned}
& \left|\int_{\mathbb{R}^{2}} \Delta_{k}(\operatorname{div}(h u)) \Delta\left(\Delta_{k} h\right) d x\right| \leq\left|\int_{\mathbb{R}^{2}} \Delta_{k}(\nabla h \operatorname{div} u) \Delta_{k}(\nabla h) d x\right| \\
& +\mid \int_{\mathbb{R}^{2}} \Delta_{k}\left(h \nabla(\operatorname{div} u) \Delta_{k}(\nabla h) d x|+| \int_{\mathbb{R}^{2}} \Delta_{k}(u \nabla h) \Delta_{k}(\Delta h) d x \mid .\right.
\end{aligned}
$$

It is easy to estimate the first and the second terms by

$$
C d_{k}^{2} 2^{-2 k \tau}\left(\|\nabla u\|_{L^{\infty}}\|\nabla h\|_{H^{\tau}}^{2}+\|\nabla h\|_{L^{2}}\|\Delta u\|_{H^{\tau}}\|\nabla h\|_{H^{\tau}}\right),
$$

while for the third term, since we cannot control $\triangle h$, it is convenient to write

$$
\begin{aligned}
& \int_{\mathbb{R}^{2}} \Delta_{k}\left(T_{u} \nabla h\right) \Delta_{k}(\triangle h) d x=\sum_{|q-k| \leq N_{1}} \int_{\mathbb{R}^{2}} \Delta_{k}\left(S_{q} u \nabla h_{q}\right) \Delta_{k}(\triangle h) d x \\
& =\sum_{|q-k| \leq N_{1}} \int_{\mathbb{R}^{2}}\left(\left(S_{q} u \Delta_{k} \Delta_{q}(\nabla h)\right)+\left[\Delta_{k}, S_{q} u\right] \Delta_{q}(\nabla h)\right) \Delta_{k}(\triangle h) d x \\
& =\sum_{|q-k| \leq N_{1}} \int_{\mathbb{R}^{2}}\left(\left(S_{q}-S_{k}\right) u \Delta_{k} \Delta_{q}(\nabla h)+\left[\Delta_{k}, S_{q} u\right] \Delta_{q}(\nabla h)\right) \Delta_{k}(\triangle h) d x \\
& \quad+\int_{\mathbb{R}^{2}} S_{k} u \nabla\left(\Delta_{k} h\right) \triangle\left(\Delta_{k} h\right) d x=K_{1}+K_{2}+K_{3} .
\end{aligned}
$$


Since $\left(S_{q}-S_{k}\right) u=-\sum_{q \leq p \leq k-1} \Delta_{p} u$

$$
\begin{aligned}
K_{1} & \leq C \sum_{p}\left\|\Delta_{p} u\right\|_{L^{\infty}}\left\|\nabla \Delta_{k} h\right\|_{L^{2}}\left\|\Delta \Delta_{k} h\right\|_{L^{2}} \\
& \leq C d_{k}^{2} 2^{-2 k \tau}\|\Delta u\|_{H_{\tau}}\|\nabla h\|_{L^{2}}\|\nabla h\|_{H^{\tau}} .
\end{aligned}
$$

As in the proof of lemma 5.1, we have

$$
K_{2} \leq C d_{k}^{2} 2^{-2 k \tau}\|\nabla u\|_{L^{\infty}}\|\nabla h\|_{H^{\tau}}^{2}
$$

Then, using the following computation

$$
\begin{aligned}
\int_{\mathbb{R}^{2}}\left(S_{k} u\right. & \left.\nabla\left(\Delta_{k} h\right)\right) \triangle\left(\Delta_{k} h\right) d x \\
& =\int_{\mathbb{R}^{2}}\left(\frac{1}{2} \operatorname{div}\left(S_{k} u\right)\left|\nabla\left(\Delta_{k} h\right)\right|^{2}-\sum_{i, j} \partial_{j}\left(S_{k} u^{i}\right) \partial_{i}\left(\Delta_{k} h\right) \partial_{j}\left(\Delta_{k} h\right)\right) d x
\end{aligned}
$$

we get immediately that

$$
K_{3} \leq C d_{k}^{2} 2^{-2 k \tau}\|\nabla u\|_{L^{\infty}}\|\nabla h\|_{H^{\tau}}^{2},
$$

and this proves the Lemma.

\section{References}

[1] Bony, J.-M.: Calcul symbolique et propagation des singularités pour les équations aux dérivées partielles non linéaires. Ann. Sci. École Norm. Sup. (4) 14 (1981), no. 2, 209-246.

[2] Bui, A. T.: Existence and uniqueness of a classical solution of an initial boundary value problem of the theory of shallow waters. SIAM J. Math. Anal. 12 (1981) 229-241.

[3] Chemin, J.-Y.: Fluides parfaits incompressibles. Astérisque 230 (1995).

[4] Chemin, J.-Y., Desjardins, B., Gallagher, I. and Grenier, E.: Fluids with anisotropic viscosity. Special issue for R. Temam's 60th birthday. M2AN Math. Model. Numer. Anal. 34 (2000), no. 2, 315-335.

[5] Kloeden, P. E.: Global existence of classical solutions in the dissipative shallow water equations. SIAM J. Math. Anal. 16 (1985), 301-315.

[6] LiU, T.-P. And Wang, W.K.: The pointwise estimates of diffusion wave for the Navier-Stokes systems in odd multi-dimensions. Comm. Math. Phys. 196 (1998), 145-173.

[7] Matsumura, A. And Nishida, T.: The initial value problem for the equations of motion of viscous and heat-conductive gases. J. Math. Kyoto Univ 20 (1980), 67-104. 
[8] Matsumura, A. And Nishida, T.: Initial-boundary value problems for the equations of motion of general fluids. In Computing methods in applied sciences and engineering, V (Versailles, 1981), 389-406. North-Holland, Amsterdam, 1982.

[9] Matsumura, A. And Nishida, T.: The initial value problem for the equations of motion of compressible viscous and heatconductive fluids. Proc. Japan Acad. Ser. A Math. Sci. 55 (1979), no. 9, 337-342.

[10] Sundbye, L.: Global existence for Dirichlet problem for the viscous shallow water equations. J. Math. Anal. Appl. 202 (1996), 236-258.

[11] Sundbye, L.: Global existence for the Cauchy problem for the viscous shallow water equations. Rocky Mountain J. Math. 28 (1998), 1135-1152.

[12] Wang, W-K. And Yang, T.: The pointwise estimates of solutions of Euler equations with damping in multi-dimensions. J. Differential Equations $\mathbf{1 7 3}$ (2001), 410-450 .

Recibido: 14 de Febrero de 2002

\author{
Weike Wang \\ Department of Mathematics \\ Shanghai Jiao Tong University \\ 200030 Shanghai \\ China \\ wkwang@sjtu.edu.cn \\ Chao-Jiang $\mathrm{Xu}$ \\ Mathématiques UMR 6085 \\ Université de Rouen \\ 76821 Mont Saint Aignan \\ France \\ Chao-Jiang. Xu@univ-rouen.fr
}

\footnotetext{
The present work was carried out when the first author was in a visiting post of CNRS at Université de Rouen, the laboratoire de mathématiques Raphaël Salem UMR 6085. Their work was also supported in part by National Natural Science Foundation of China 10131050.
} 